\section{Parent-mediated Training for Children with Autism Spectrum Disorder in India}

In India, prevalence of autism spectrum disorder (ASD) is estimated to be $1-1.4 \%$ ( 1 in 100 children) in children aged $2-9$ years [1]. However, there continues to be a deficit of trained therapists to address these children [2]. Parent-implemented interventions with adequate guidance from trained therapists could be the best alternative for young ASD children [3]. We have developed an online intervention program (SCoPEEDITT: Socio Communication Play and Educational ProgramEducating Parents on Direct and Interactive Teaching Techniques) to involve and support parents in the early intervention of children with ASD (or at risk for ASD). Parentimplemented intervention also extends the benefits of intervention to the home environment thereby supporting in generalization.

The purpose of this pilot study was to find whether the short term parental training brings about changes in parental interactive behavior and child engagement skills and also to assess effectiveness of the SCoPE program. Participants were 22 young children (Mean age 27.3 months) with diagnosis of $\mathrm{ASD} / \mathrm{at}$ risk for ASD. Before beginning the intervention, baseline data on children's language and cognitive skills was assessed using Mullen Scale of Early Learning (MSEL). Parents were asked to play and interact with their child in their usual way for $10 \mathrm{~min}$ in the centre and it was videotaped. Parents' baseline interactive behavior and children's engagement skills were observed and rated using Modified Maternal Behaviour Rating Scale (MBRS) and Modified Child Behaviors Rating Scale (CBRS) [7]. CBBS identiûes two domains (attention domain and initiation domain) of children's interactive engagement with their parents. In our study, CBRS scale was modified by excluding affect and persistence items.

Parents were trained over 4 weeks to learn and use interactive parenting techniques with structured EDITT program and suggested to use individualized SCoPE based activities during homework sessions. All children continued regular weekly (weekly twice for 45 minutes) language therapy sessions (with/without sensory integration sessions based on individual child requirement) with trained therapists. Parents and their children were followed up for next 9 months. After 1 month of EDITT parental training program, parents showed significant improvement in all the interactive behaviors, and decline in directive behaviour parameters of MBRS. At 3 months follow up, parents showed significant improvement in their interactive behavior and further decline in directive behaviors (Sensitivity $\mathrm{d}=4.87$, Responsiveness $\mathrm{d}=3.78$, Effectiveness $\mathrm{d}=3.37$, Achievement $\mathrm{d}=3.78$, Reinforcing behaviour $\mathrm{d}=3.08$ and Directiveness $\mathrm{d}=-2.74$ ) with maximum improvement in their sensitivity towards their children.

At 1 month and 3 months follow up, children showed significant improvement in their engaging skills in all the parameters of Modified CBRS, Attention $\mathrm{d}=4.16$, Cooperation $\mathrm{d}=2.8$, Involvement $\mathrm{d}=2.5$, Initiating behaviors $\mathrm{d}=1.94$ ). Children showed maximum improvement in their attention behavior and least improvement in their initiating behavior.

At 9 months follow up, children showed significant improvement $(P<0.01)$ in language and cognitive domains of MSEL. On average, children showed 12.3 months improvement in receptive language, 10.7 months improvement in expressive language and 9.6 months improvement in cognitive domain over 9 months of SCoPE based intervention. $66 \%$ children showed improvement equivalent or more than 9 months in all domains.

As per existing scientific evidence, active involvement of caregivers with appropriate supervision and training as a form of co-therapy is desirable but there is not much evidence for the efficacy of parent-mediated approaches [4]. Our study adds evidence to efficacy of parent mediated approach. SCoPEEDITT program not only empowered the parents to serve as co therapists but also facilitated joint attention, imitation skills, language and cognitive development in the children with ASD.

Acknowledgement: Dr Akhila Nagaraj, Developmental Pediatrician, Centre for Child Development and Disabilities.

Kirthika Rajaraman, * Nandini Mundkur Center for Child Development and Disabilities, Bengaluru, Karnataka, India. *rkirthi83@gmail.com

\section{REFERENCES}

1. Arora NK, Nair MKC, Gulati S, et al. Neurodevelopmental disorders in children aged 2-9 years: Population-based burden estimates across five regions in India. PLoS Med. 2018; 15: e1002615.

2. Mahapatra P, Pati S, Sinha R, et al. Parental care-seeking pathway and challenges for autistic spectrum disorders children: A mixed method study from Bhubaneswar, Odisha. Indian J Psychiatry. 2019;61:37-44.

3. Zwaigenbaum L, Bauman ML, Choueiri R, et al. Early intervention for children with autism spectrum disorder under 3 years of age: Recommendations for practice and research. Pediatrics. 2015; 136:S60-81.

4. Mahoney G, Kim J, Lin C. Pivotal behavior model of developmental learning. Infants Young Child. 2007;20:311-25.

5. Oono IP, Honey EJ, McConachie H. Parent mediated early intervention for young children with autism spectrum disorders (ASD). Evid Based Child Health. 2013;8: 2380-479. 